\title{
A equipe multiprofissional da 'Saúde da Família': uma reflexão sobre o papel do fisioterapeuta
}

\author{
'Family health' multiprofessional teams: \\ a reflection on the physiotherapist's role
}

M ônica de Rezende ${ }^{1}$

$M$ arcelo Rasga M oreira ${ }^{2}$

Antenor Amâncio Filho ${ }^{1}$

Maria de Fátima Lobato Tavares ${ }^{1}$

${ }^{1}$ Departamento de

Administração e

Planejamento em Saúde,

Escola Nacional deSaúde

Pública Sérgio Arouca,

Fundação O swaldo Cruz

RuaLeopoldo Bulhões

1480/707, M anguinhos.

21041-21 Rio deJaneiro RJ.

monicarezende@ensp.fiocruz.br

${ }^{2}$ Departamento de Ciências

Sociais, Escola Nacional de

Saúde Pública Sérgio

Arouca, Fundação Oswaldo

Cruz.
Abstract This article is intended to contribute for the debate instituted about the composition of "Family $\mathrm{H}$ ealth" teams. It must be understood as a reflection about the possibility of integrating the physiotherapist to those teams, aiming to present some aspects of the profession that might potentially improve the outcomes of primary health care. On this study, weanalyzethelegal documentsthat approvetherules for qualifying professional physiotherapists. In the 1970sand 1980s, following Brazilian sanitary reform, those documents made official the process that led physiotherapy, which was historically recognized for its clinic acting, into a shift on its work object. This change approximated physiotherapists to preventive and health promotion practices, typical of the first level of care. We conclude that such changes opened an important spacefor integration still little explored. We propose to strengthen these new fields, by focusing the reflection on integration forms and on the necessity to impose to managers to plan their actions in association with the community, taking into account the local health situation and assuring intervention on problems according to priority population groups.

Key words Family Health, Physiotherapy, Multiprofessional teams
Resumo Este artigo pretende contribuir para 0 debate instituído sobre a composição das equipes de Saúde da Família. Deve ser apreendido como uma reflexão sobrea possi bilidade de integração do fisioterapeuta nas equipes, na intenção de mostrar os aspectos da profissão que a tornam capaz de potencializar a resolutividade da atenção básica. No estudo, foram analisados os documentos legais que aprovam as normas para habilitação ao exercício da profissão de fisioterapeuta. Nos anos setenta e oitenta, acompanhando a reforma sanitária brasileira, tais documentos oficializaram o processo que levou a fisioterapia, profissão historicamente reconhecida pela atuação clínica, a mudar seu objeto de trabalho, aproximando o fisioterapeuta de práticas preventivas e de promoção da saúde, típicas do primeiro nível do cuidado. Concluiu-se que tal mudança abriu importante espaço de integração ainda pouco trabal hado e propõe-se seu aprofundamento, direcionando a reflexão para as formas de integração ea necessi dade que se impõe aos gestores de planejar suas ações deforma articulada com a comunidade, a partir da análise da situação de saúde no nível local, garantindo a intervenção sobre problemas e grupos populacionais prioritários. Palavras-chave Saúde da Família, Equipes multiprofissionais, Fisioterapia 


\section{Introdução}

O Sistema Único de Saúde(SU S), resultado de um processo de lutas políticas e setoriais capitaneadas pelo movimento sanitário brasileiro, tem o objetivo de garantir a saúde como direito do cidadão edever do Estado. Propõe, como horizonte, a superação dos limites do modelo médico-assistencial privatista, que tem como bases a especialização do profissional e a ênfase na doença e no processo de cura.

Nessa dimensão, a incorporação do conceito ampliado de saúde, que a associa às condições de vida, norteia a formulação e a implementação de estratégias que viabilizem um serviço de saúdeuniversal, integral, eficaz, eficiente, com equidade e participação popular.

Uma dessas estratégias é a "Saúde da Família". Inserida no nível da atenção básica do sistema, fundamenta-se no trabalho de equipes multiprofissionais que, de acordo com o M inistério da Saúde, devem ser compostas, no mínimo, por um médico, um enfermeiro, um auxiliar de enfermagem e seis agentes comunitários de saúde e, quando amplia$\mathrm{da}$, inclui ainda um dentista, um auxiliar de consultório dentário eum técnico em higiene dental ${ }^{1}$.

A co-responsabilidade dos governos municipais e federal pelo financiamento das equipes de Saúde da Família, somada à diretriz apresentada pelo Ministério da Saúde para sua composição mínima, cria a necessidade de ampliar a discussão sobre a integração, nas equipes, de outras categorias profissionais não contempladas diretamente na proposta do governo federal, assim como sobre as possíveis formas de se fazer essa integração.

Formular e implementar um sistema de saúde estruturado sob os princípios da universalidade, da integralidadee da equidade, ten do como diretrizes a descentral ização da gestão, a regionalização e hierarquização do planejamento e oferta dos serviços desaúdeea participação comunitária (controle social), implica, necessária e obrigatoriamente, radical mudança de concepção, de mentalidade no tocante à compreensão da saúde e sua dimensão e, consequentemente, em relação ao fenômeno vida.

A demanda por reflexão e diálogo em torno dessa perspectiva nova ocorre tanto na esfera interna de cada profissão quanto no campo da saúde como um todo. Por um lado, é preciso que os profissionais reflitam conjuntamente sobre as finalidades de sua prática, reconstruindo o sentido e o significado do seu agir, tornando-os coerentes com a proposta apresentada, em especial no que tange à atenção básica. Por outro, torna-se indispensável a ponderação, junto a gestores e organizações comprometidas com a participação social, sobre a relação entre a composição das equipes e as necessidades de saúde das comunidades.

Desse modo, identificar os objetos de trabal ho e estudo da fisioterapia por intermédio da análise dedocumentos legais queaprovam as normas para habilitação ao exercício da profissão de fisioterapeuta; relacionar as mudanças destes objetos no decorrer do processo de construção do Sistema Único de Saúde com as transformações ocorridas no cenário da Saúde Pública no Brasil eanalisar de que forma a fisioterapia pode, a partir destas mudanças, integrar-se à estratégia Saúde da Família, são propostas que se encontram contidas e permeiam este artigo.

0 presente artigo, pois, se insere no contexto sanitário atual como uma contribuição ao debate instituído sobre a composição das equipes de Saúde da Família. Deve ser apreendido como uma reflexão cujos objetivos se assentam na análise sobre a possibilidade de integração do fisioterapeuta nas equipes de Saúde da Família, na intenção de mostrar os aspectos da profissão que a tornam capaz de potencializar a resolutividade nesse primeiro nível da atenção à saúdeena expectativa de se constituir em instrumento para o fortalecer a estratégia Saúde da Família, somando esforços na direção da consolidação dos princípios e pressupostos do Sistema Único de Saúde brasileiro.

Importante registrar, também, que este texto é resultado de estudo realizado no âmbito da linha de pesquisa que abriga o projeto "Avaliação da inserção do fisioterapeuta na estratégia Saúde da Família: um estudo de caso". Tal projeto foi aprovado e selecionado de acordo com os termos do Edital no 49/2005, iniciativa conjunta dos M inisté rios da Saúde e da Ciência eTecnologia. Foi desenvolvido pela Escola N acional deSaúde Pública Sergio Arouca, da Fundação Oswaldo Cruz, no município de Macaé, Estado do Rio de Janeiro, no período de 2005 a 2007, com o apoio do Conselho Nacional de Desenvolvimento Científico e Tecnológico (CN Pq) e colaboração da Secretaria Municipal de Saúde do citado município.

\section{Oscaminhos dafisioterapia}

A fisioterapia surgiu como uma "especialidade paramédica ${ }^{2 "}$ com o propósito de reabilitar e preparar pessoas fisicamente lesadas nas grandes guerras, em acidentes detrabal ho ou por doenças oriundas das condições sanitárias precárias para o retorno à vida produtiva ${ }^{3}$.

No Brasil, foi criada como profissão de nível superior em outubro de 1969, pelo Decreto-Lei no. 
938, que, em seu art. 3o, estabeleceu que "éatividade privativa do fisioterapeuta executar métodos e técnicas fisioterápicas com a finalidade de restaurar, desenvolver e conservar a capacidade física do paciente ${ }^{4 \prime}$.

0 uso do termo "paciente" indica uma proposta de atuação voltada para o indivíduo doente e, portanto, já em fase de tratamento ${ }^{3}$. É coerente com o pensamento clínico sobre o processo saúde-doença, que faz com que a saúde seja "entendida ou representada como ausência de doença e a organização dos serviços medicamente definida5".

Fundamentados neste ideário, os esforços dos profissionais e/ou áreas de estudo preocupadas com as condições de saúde do homem concentram-se na descoberta de novos métodos de "tratamento" das doenças, revelando a predominância de uma assistência "curativa", recuperativa ereabilitadora.

$\mathrm{N}$ a década de setenta, surgiram, no cenário mundial, novos conceitos de saúdee novas concepções do processo saúde-doença mais vinculadas à qualidade de vida de uma população e que procuravam articular, como dimensões explicativas, a biologia humana, os estilos de vida, o ambiente e serviços de saúde. A publicação do documento A new perspective on the health of Canadians, em 1974, por $M$ arc Lalonde 6 , marca 0 início do moderno movimento da promoção da saúde e apresenta a perspectiva da história social das doenças.

A partir da Declaração de Alma-Ata, em 1978, a Atenção Primária à Saúde (APS) passou a ser defendida como estratégia para se al cançar a meta "Saúde para Todos", inicialmente proposta com o ano 2000 como prazo limite, posteriormente estendido para o século XXI. De acordo com a proposta, governos, organizações internacionais etoda a comunidade mundial deveriam unir esforços para que todos os povos do mundo atingissem um nível de saúde que lhes permitissem levar uma vida social e economicamente produtiva?.

No Brasil, acompanhando esse movimento, intensificaram-seas críticas ao modelo vigente(centrado na assistência médico-hospitalar), resultando na implantação, por intermédio da reforma do ensino médio e da saúde pública ${ }^{8}$, da proposta da medicina preventiva.

Em 1975, com a criação do Conselho Federal de Fisioterapia e Terapia Ocupacional (COFITTO), pela Lei $n-6.316$, iniciou-se o processo de regulamentação da profissão de Fisioterapia. Tendo 0 Consel ho, como uma de suas incumbências, exercer função normativa, emitiu, em fevereiro de 1978, a Resolução COFITTO no 08, aprovando as normas para habilitação ao exercício da profissão de fisioterapeuta que, refletindo o movimento da saúdeentão vigente, definiu como atos desse profissional, "planejar, programar, ordenar, coordenar, exe cutar e supervisionar métodos e técnicas fisioterápicos que visem à saúde nos níveis de prevenção primária, secundária e terciária".

Em relação ao Decreto-Lei no 938/69, a Resolução COFITTO no 08/78 ampliou consideravelmente o campo de atuação do fisioterapeuta, tanto em relação aos níveis de assistência (prevenção primária, secundária e terciária) quanto ao foco da atenção, passando a apreender a saúde do indivíduo como um todo e não mais apenas no que diz respeito à sua capacidade física.

Estas alterações conceituais, embora importantes, demonstram-se frágeis quando a mencionada Resolução, no seu Art. 3ㅇ, fixa 0 ato profissional na terapia física: "prescrever, ministrar e supervisionar terapia física que objetive preservar, manter, desenvolver ou restaurar a integridade de órgão, sistema ou função do corpo humano ${ }^{9 \prime}$ [ grifo nosso], posto que o termo "terapia" está diretamenterelacionado ao substantivo "terapêutica" que se refere à "parte da medicina que estuda e põe em prática os meios adequados para aliviar ou curar os doentes ${ }^{10 \prime}$.

A partir do momento em que os fisioterapeutas ampliaram seu campo de atuação e apresentaramse como aptos a contribuir, também, na prevenção primária, eles criaram a necessidade de rever ereestruturar sua prática. 0 processo de transformação, no entanto, ficou incompleto, na medida em que faltou atribuir a si, na regulamentação, ações de educação em saúde e prevenção de doenças.

A década de oitenta foi um período efervescente, em que o M ovimento da Reforma Sanitária despontou como um dos protagonistas políticos na luta pela redemocratização do país. Respaldado pelas novas concepções do processo saúde-doença, surgidas no cenário mundial, o movimento buscou a redefinição da política de saúde brasileira, a partir da incorporação de seus determinantes sociais, da ênfase às condições de vida e da garantia do direito do exercício da cidadania.

A Oitava Conferência Nacional de Saúde (8a CNS), cuja realização esteve diretamente vinculada ao movimento sanitarista, ocorreu em março de 1986, tendo como um de seus principais temas (senão o principal), a reformulação do sistema nacional de saúde. Em seu relatório final ${ }^{11}$, a saúde foi definida em sentido mais abrangente, como "resultado das formas de organização social da produção, as quais podem gerar grandes desigualdades nos níveis de vida" e foi proposta a criação de um sistema unificado de saúde, com "a integra- 
lização das ações, superando a dicotomia preventivo-curativo".

Em maio de 1987, após a 8a CNS e antes da criação do Sistema Ú nico de Saúde, a Resolução COFFITO no 80, por meio de atos complementares, buscou ampliar as atribuições do fisioterapeuta expressas na Resolução no 08/78, numa perspectiva que procurou adequar a Fisioterapia ao novo momento do cenário sanitário brasileiro.

Dentre as considerações da Resolução $n=80$, deve ser destacada a que alude ao objeto de estudo e trabalho do fisioterapeuta: A fisioterapia é uma ciência aplicada, cujo objeto de estudos éo movimento humano em todas as suas formas de expressão e potencialidades, quer nas suas alterações patológicas, quer nas suas repercussões psíquicas e orgânicas, com objetivos de preservar, manter, desenvolver ou restaurar a integridade de órgão, sistema ou função $0^{12}$.

Essa foi uma mudança essencial, porque 0 movimento é a forma de comunicação do indivíduo com o mundo externo, que acontece a partir da sua interação com o meio em que vive. Não há comunicação sem movimento: a fala produz-se a partir do movimento; a respiração só ocorre com movimento; através do movimento, os indivíduos expressam seus sentimentos e assumem posturas diante dos desafios da vida. Assim, ao assumir o movimento como seu objeto, o fisioterapeuta adotou uma perspectiva relacional, pensando o sujei to não apenas por suas características biológicas, mas também o considerando na sua dimensão social.

Visto dessa forma, o fisioterapeuta deve atuar em interação com outros profissionais, para me Ihor compreender e poder interferir positivamente, no sentido de ampliar e de tornar mais rica e prazerosa a relação permanente do indivíduo com seu ambiente. Junto com os usuários dos serviços de saúde, deve refletir sobre o uso que eles fazem do próprio corpo nas suas relações com o espaço e com outras pessoas e, a partir de então, promover ações visando à melhoria da qualidade de vida no presente e para o futuro. Muito mais do que tratar e reabilitar, o fisioterapeuta tem o encargo de agir na direção do desenvolvimento das potencialidades do indivíduo para exercer suas atividades laborativas e da vida diária. Esse posicionamento está diretamente relacionado ao que explicita a Resolução COFITTO no 80/87: "Por sua formação acadêmico-profissional, pode o fisioterapeuta atuar juntamente com outros profissionais nos diversos níveis da assistência à saúde, na administração de serviços, na área educacional e no desenvolvimento de pesquisas ${ }^{12 "}$.

Esta consideração atesta o fisioterapeuta como um profissional apto a atuar na aten ção básica tal como se configura na atualidade: o primeiro nível do sistema de assistência à saúde, articulado com os demais, com o intuito de garantir maior acesso aos cuidados e integralidade da atenção, consoante a Declaração de Alma-Ata de que os cuidados primários "têm em vista os problemas de saúde da comunidade, proporcionando serviços de promoção, prevenção, cura e reabilitação, conforme as necessidades ${ }^{\prime \prime}$.

A reabilitação, a partir dos anos oitenta, assumiu perspectiva diferente da adotada na medicina preventiva, que se encontra respaldada na história natural das doenças. 0 documento "Programa de Ação Mundial para Pessoas com D eficiência", aprovado edivulgado pela O rganização das Nações Unidas em 1982, ressaltou que o direito dos "deficientes" (termo utilizado no documento) situava-se no mesmo patamar de oportunidades dos demais cidadãos, ao afirmar que a eles também caberia "usufruir, em condição de igualdade, das melhorias nas condições de vida". A novidade decorreu de uma visão abrangentee multidimensional, que compreendeu ações dereabilitação como treinamento eempowerment do portador de necessidades especiais, intervenções sociais amplas, adaptação do meio ambiente e proteção dos direitos do portador ${ }^{13}$.

A discussão conceitual em relação ao portador de necessidades especiais e à reabilitação levou à associação de dois componentes principais: o funcional eo social. Esteenfoqueinfluenciou, também, a assistência em reabilitação daqueles que apresentam lesões transitórias e foi referida em uma das considerações da citada Resolução no 80/87, que apresentou a reabilitação como "uma proposta de atuação multiprofissional voltada para a recuperação e o bem-estar bio-psico-social do indivíduo 12".

Afora a discussão do objeto do fisioterapeuta, a possibilidade de trazer a reabilitação e 0 tratamento de distúrbios do movimento para o ambiente em que o indivíduo vive e desenvolve suas atividades - tornando-os mais eficientes, acessíveis e prevenindo transtornos maiores para as pessoas que convivem com algum problema - ratifica, sobremodo, o espaço que se abre para a Fisioterapia no primeiro nível da atenção.

Contudo, de acordo com os termos da Resolução $\mathrm{n}$ 0 80/87, 0 ato do fisioterapeuta é, ainda, restrito ao "processo terapêutico" e, portanto, à relação com a doença: "Baseando-se nas condições psico-físico-social, [o fisioterapeuta] busca promover, aperfeiçoar ou adaptar, através de uma relação terapêutica, o indivíduo a uma melhor qualidade de vida ${ }^{12 "}$.

Para melhor entendimento da questão, cabe assinalar a relação observada entre transforma- 
ções marcantes no âmbito da Fisioterapia e mudanças importantes ocorridas na área da saúde, vista como um todo. Nesse sentido, o Quadro 1 sintetiza um conjunto de eventos, os quais informam e subsidiam a construção do presente texto.

O desenho comparativo apresentado no Quadro 1 permite perceber as mudanças no objeto de estudo, no campo de atuação e no foco da atenção da fisioterapia, em consonância com as alterações que ocorreram, no mesmo período, na área da saúde. Entretanto, dois elementos suscitam reflexão: 1) 0 ato profissional permaneceu enfatizando a terapia física e, 2) após a criação do Sistema Ú nico de Saúde, não houve nenhuma complementação a essas resoluções.

A insistência no ato terapêutico, enquanto uma intervenção destinada a minorar ou solucionar um problema físico localizado, parecesintomática. Para esse tipo de atividade, o fisioterapeuta encontra mercado de trabalho e reconhecimento profissional. Vale considerar que, embora a Fisioterapia seja recentecomo profissão, apresenta-secomo um dos campos mais antigos da saúde, pois os agentes físicos vêm sendo utilizados há milhares de anos no tratamento das patologias, sendo que determinadas condutas fisioterapêuticas construídas em tor- no desse eixo "tem mostrado inegável eficácia nas diversas áreas em que atua ${ }^{14 "}$ e no enfrentamento de variadas situações.

0 segundo elemento de reflexão diz respeito à inserção e ao papel do fisioterapeuta no Sistema Ú nico de Saúde, criado como parte de uma dinâmica histórica esocial que significa um olhar diferente em relação à percepção sobre as causas das doenças e dos problemas de saúde. A nova política sanitária obriga, em especial, mudanças nos modelos de atenção, definidos genericamente como "combinações de saberes (conhecimentos) etécnicas (métodos einstrumentos) utilizados para atender necessidades de saúdeindividuais e coletivas ${ }^{15 "}$.

No entanto, mudar modelos instalados e em operação é um processo lento e tenso, na medida em que eles representam políticas e práticas que retratam interesses profissionais, de instituições e de corporações. A tensão tem reflexo e encontra ressonância nas decisões (ou ausência delas) de cada categoria profissional. No tocante à Fisioterapia, se evidencia pela ausência de complementação da regulamentação da profissão pós surgimento do Sistema Ú nico de Saúde, principalmenteem relação ao ato profissional.

Quadro 1. Relação entre as transformações na regulamentação da fisioterapia e na área da saúde.

\begin{tabular}{|c|c|c|}
\hline Décadas & Área da Saúde & Fisioterapia \\
\hline 1960 & $\begin{array}{l}\checkmark \text { Predomínio do pensamento clínico sobre } \\
\text { o processo saúde-doença; } \\
\checkmark \text { Paradigma flexneriano. }\end{array}$ & $\begin{array}{l}\text { Decreto-Lei no } 938 \text { (1969) } \\
\checkmark \text { Cria a profissão de fisioterapeuta; } \\
\checkmark \text { Ato profissional: terapia física (tratamento); } \\
\checkmark \text { Foco da atenção: capacidade física. }\end{array}$ \\
\hline 1970 & $\begin{array}{l}\checkmark \text { Surgimento de novos conceitos de saúde } \\
\text { e de novas concepções do processo saúde- } \\
\text { doença; } \\
\checkmark \text { Sistemas de saúde dos países } \\
\text { desenvolvidos fortemente questionados; } \\
\checkmark \text { No Brasil: implantação da proposta da } \\
\text { medicina preventiva. }\end{array}$ & $\begin{array}{l}\text { Resolução COFITTO no } 08 \text { (1978) } \\
\checkmark \text { Objeto de estudos: corpo humano (órgão, } \\
\text { sistemas e funções); } \\
\checkmark \text { Campo de atuação: prevenção primária, } \\
\text { secundária e terciária; } \\
\checkmark \text { Ato profissional: terapia física; } \\
\checkmark \text { Foco da atenção: saúde. }\end{array}$ \\
\hline 1980 & $\begin{array}{l}\checkmark \text { M ovimento da Reforma Sanitária; } \\
\checkmark \text { "Programa de Ação M undial para Pessoas } \\
\text { com Deficiência" / ONU (1982): Visão } \\
\text { abrangente e multidimensional da } \\
\text { reabilitação. } \\
\checkmark \text { VIII CNS (1986); } \\
\checkmark \text { Criação do SUS (1988). }\end{array}$ & $\begin{array}{l}\text { Resolução COFITTO no } 80 \text { (1987) } \\
\checkmark \text { Objeto de estudos: movimento humano; } \\
\checkmark \text { Campo de atuação: diversos níveis da } \\
\text { assistência à saúde; } \\
\checkmark \text { Ato profissional: terapia física; } \\
\checkmark \text { Foco da atenção: saúde (qualidade de vida e } \\
\text { bem-estar biopsicossocial do indivíduo). }\end{array}$ \\
\hline
\end{tabular}

Fonte: Elaborado pelos autores. 


\section{A busca pela atenção integral à saúde}

0 fundamento para a regionalização dos serviços e dos sistemas de serviços de saúde organizados em bases populacionais, juntamente com a moderna concepção de atenção primária à saúde, é encontra-se no Relatório Dawson, de 1920, publicado no Reino Unido. No mesmo relatório, preconiza-se a organização do sistema de serviços de saúde em três níveis: os centros primários deatenção, os centros secundários e os hospitais de ensino ${ }^{16}$.

A determinação e abrangência da atenção primária à saúde como doutrina universal, entretanto, ocorreu somenteem 1978, na Conferência Internacional sobre Cuidados Primários de Saúde, realizada em Alma-A ta, a partir da qual a O rganização M undial de Saúde começou a estabel ecer estratégias para operacionalizar as metas então acordadas ${ }^{16}$.

Dessa maneira, segundo a Declaração deAlmaAta, os cuidados primários de saúde representam o primeiro nível de contato dos indivíduos, da família e da comunidade com o sistema nacional de saúde, através do qual os cuidados de saúde são levados o mais proximamente possível aos lugares onde pessoas vivem e trabalham, e constituem o primeiro ele mento de um continuado processo de assistência à saúde ${ }^{7}$.

O Sistema Ú nico deSaúde propõequeos serviços de saúde sejam organizados por níveis de atenção. A Lei 8.080, de setembro de 1990, que regula as ações e serviços de saúde no âmbito do Sistema, prevê que as ações devem ser desenvolvidas e organizadas em uma rede de serviços regionalizadae hierarquizada. A tecnologia utilizada nas ações deve ser adequada a cada nível, com mecanismos formalizados de referência e contra-referência, visando ao atendimento integral da população.

A atenção básica passa, então, a ser compreendida como "porta de entrada" para o sistema de saúde, passando a ser defendida como o primeiro nível do cuidado, representado por um conjunto de ações de saúde, no âmbito individual e coletivo, que abrangem a promoção e a proteção da saúde, a prevenção de agravos, o diagnóstico, o tratamento, a reabilitação e a manutenção da saúde ${ }^{17}$.

Assim, por possuir capacidade de resolução em relação a grande parte dos problemas de saúde da população, a atenção básica, se bem estruturada, permite reduzir o fluxo de usuários para os níveis de maior densidade tecnológica, racionalizando o uso da tecnologia e dos recursos terapêuticos mais caros e possibilitando maior acesso aos cuidados. A proposta, portanto, é viabilizar, a partir do primeiro nível do cuidado, a integração dos diversos pontos de atenção à saúde, possibilitando uma assistência contínua, de qualidade, com menor custo e em lugar e tempo certos.

Em meados da década de noventa, a partir de um acúmulo de experiências implementadas por todo o país, com destaque para o Programa de Agentes Comunitários de Saúde (PACS), o Programa Saúde da Família (PSF) éadotado pelo governo federal como eixo estruturante da atenção básica no SistemaÚ nico deSaúde. H ojechancelado como estratégia Saúde da Família, o modelo apresenta, introduz einduz mudanças relevantes no processo de trabalho em saúde, como resultado da transformação das Unidades Básicas de Saúde (UBS) em Unidades de Saúde da Família (USF) ${ }^{18}$.

Ao contrário do modelo assistencial privatista, no qual o profissional médico tem presença predominante nas intervenções, ocasionando um papel secundário aos demais profissionais que compõem a força de trabalho na saúde, a Saúde da Família baseia-se, essencialmente, no trabalho das equipes ${ }^{18}$. Cabe a elas, entre outras atribuições: cadastrar todas as famílias de uma determinada região, identificar os problemas de saúde dessa população cadastrada, planejar suas ações dentro das necessidades locais, acompanhar os grupos mais vulneráveis, realizar atendimentos de qualidade (agendados previamente, por demanda espontânea nas unidades ou em visita em domicílio), desenvolver ações de educação e promoção da saúde e estabelecer vínculos de compromisso e co-responsabilidade com as pessoas da comunidade.

Tais funções devem ser desempenhadas tanto numa abordagem individual quanto coletiva. 0 profissional deve partir da premissa de que as alterações físicas ou mentais vivenciadas pel os indivíduos geram uma desarticulação de sua existência, abalando e desestruturando a dinâmica de sua família e dos demais grupos com os quais se relacionam e nos quais se encontram inseridos. É imprescindível perceber a multicausalidade dos processos mórbidos, contextualizando, sempre, o indivíduo em seu ambiente ${ }^{19}$.

Por outro lado, a relação e o vínculo dos profissionais com os membros da comunidade, usuários dos serviços, dá-se através de ações, reações e efeitos que geram novas situações e apresentam novos desafios num processo contínuo de busca por atenuar ou superar problemas e obstáculos ${ }^{20}$. Aqui revela-se a complexidade do trabal ho profissional nesse primeiro nível da atenção ${ }^{5}$, visto queo cumprimento de uma atividade integrada e cooperativa exige o desenvolvimento de um conjunto de novas competências' ${ }^{21}$.

No entanto, além de novas competências, o trabalho profissional das equipes envolve, também, 
atividadese conhecimentostécnicos queestão constituídos e situados nas esferas de diferentes categorias profissionais. Saberes bem definidos, quecompõem a "caixa de ferramentas" do trabalhador da saúde e que concebem o olhar do profissional sobre o usuário, "enquanto objeto de sua intervenção, em um processo de captura do mundo daquele e de suas necessidades ${ }^{22 " .}$

N essa perspectiva, a integração de profissionais de várias categorias nas equipes de Saúde da Família permite distintos olhares, ampliando as possibilidades inovadoras das práticas do cuidado e aumentando o potencial de resolutividade, com qualidade e sem descaracterizar a proposta original.

\section{Consideraçõesfinais}

O processo de expansão da estratégia Saúde da Família como projeto nacional tem ocorrido a partir de suporte técnico e financeiro do governo federal aos municípios, para que ampliem o número de equipes e, consequentemente, o contingente da população atendida.

Embora a diretriz do M inistério da Saúde para a composição das equipes não seja restritiva, ela tem sido co-responsável por manter ainda tímido o debate sobre a incorporação, às equipes de saúde, das denominadas "outras categorias profissionais". Essa discussão é importante para o processo de descentralização da gestão do sistema de saúde, que tem como um de seus objetivos incentivar a análise da situação de saúde no nível local, para que a intervenção sobre problemas e grupos populacionais prioritários aconteça a partir do mapeamento de danos e riscos que afetam a população.

Dessa maneira, a elaboração de planos e a organização de ações e de serviços poderiam acontecer de forma articulada, com participação da comunidade, ao abrir-se um amplo espaço de discussão para pensar, coletivamente, quais profissionais estariam mais aptos a contribuir para a meIhoria da saúde e da qualidade de vida da população de determinada região.

A proposta atual do M inistério da Saúde para a inserção do fisioterapeuta na estratégia Saúde da Famíliaéqueela se dêatravés dos Núcleos deAtenção Integral na Saúde da Família (NAISF), criados pela Portaria n-1.065/GM, de 04 de julho de 2005.

A forma como os núcleos foram estruturados tem levantado polêmica entre os profissionais que atuam na saúde, com al gumas questões sendo consideradas pontos críticos, como a implantação de um núcleo para cada nove a onze equipes (população maior ou igual a 40 mil habitantes). N o caso particular do tema central deste artigo, a crítica volta-se para a visão restrita quanto à função do fisioterapeuta. A inserção deste profissional através dos núcleos, da forma como proposta, limita à reabilitação a contribuição que o fisioterapeuta pode oferecer ao cuidado no nível da atenção básica, na Saúde da Família.

O que este artigo propõe é pensar novas formas de integrar o fisioterapeuta às equipes, com suas atribuições indo além da atividade de reabilitação, fazendo com que el ese envolva e se comprometa com ações de promoção e proteção da saúde, de prevenção de doenças e de assistência. $M$ ais do que inserir o fisioterapeuta na Saúde da Família, aproximando-o da atenção básica, sua integração às equipes compreende a idéia de criar pontos de interseção, tanto nas ações realizadas como entre os profissionais, facilitando e incentivando a adoção de medidas que conformem um olhar e uma prática integral da saúde.

A integração proposta depende de diversos fatores, fundamentados no reconhecimento do valor agregado com esta participação. Dentre eles, inclui-se a reflexão sobre o "o que fazer" da profissão na Saúde da Família, o que torna relevante e necessário rever a formação dos fisioterapeutas, tendo em vista que, muitas vezes, estão reproduzindo práticas sem refletir sobre elas e sobre o papel que elas ocupam e desempenham na dinâmica social, política, econômica e cultural do país.

Contudo, apesar das dificuldades encontradas até o momento, alguns municípios já incluíram o fisioterapeuta nas suas equipes de Saúde da Família. Tais vivências precisam ser observadas e analisadas. Estudos e investigações sobre esse fenômeno, buscando identificar as peculiaridades e especificidades decada situação enfocada, precisam ser disseminados, gerando, inclusive, informações e dados que subsidiem a tomada de decisões que fortaleçam e criem inovações no processo de planejamento, gestão e educação na saúde.

\section{Colaboradores}

M Rezendeidealizou eplanejou o artigo, o levantamento dos dados, a redação preliminar; M R M oreira contribuiu ao planejamento e análise crítica do texto; A Amâncio Filho e M FL Tavares contribuíram para a formulação de questões, redação e revisão final do texto. Todos participaram da análise dos dados e da discussão dos resultados. 
1. Brasil. Ministério da Saúde. Atenção Básica: Saúde da Família. [site na Internet]. [acessado 2006 ago 3]. Disponível em: http://dtr2004.saude.gov.br/dab

2. Dicionário Houaiss da língua portuguesa. Rio de Janeiro: Objetiva; 2001.

3. Rebelatto JR, Botomé SP. Fisioterapia no Brasil: fundamentos para uma ação preventiva e perspectivas profissionais. São Paulo: Manole; 1999.

4. Brasil. Decreto-Lei 938. Provê sobre as profissões de Fisioterapeuta e Terapeuta Ocupacional, e dá outras providências. Diário Oficial da União 1969; 14 out.

5. Mendes EV. U ma agenda para a saúde. São Paulo: Hucitec; 1996.

6. Lalonde M. A new perspective on the health of Canadians: a working document. Ottawa: Minister of Supply and Services; 1974.

7. World Health Organization. Declaration of Alma-Ata. International Conference on Primary Health Care. Alma-Ata: World Health Organization; 1978.

8. Sabroza PC. Concepções de saúde e doença. Rio de Janeiro: Escola Nacional de Saúde Pública Sérgio Arouca, Fundação Oswaldo Cruz; 2004. [M imeo].

9. Brasil. Resolução COFFITO $\mathrm{n} 008$. Aprova as normas para habilitação ao exercício da profissão de fisioterapeuta e terapeuta ocupacional e dá outras providências. Diário Oficial da União 1978; 13 nov.

10. Ferreira $A B H$. Novo Dicionário Eletrônico Aurélio. Versão 5.11a. Curitiba: Positivo informática Ltda; 2004.

11. Chorny AH, Sampaio AC, Duarte A, Costa AM, Pederneiras $A R$, Pedrosa $N$ eto $A H$ et al. [relatores]. Relatório Final da 8a Conferência Nacional de Saúde. In: Anais da 8a Conferência Nacional de Saúde. Brasília: Ministério da Saúde, 1987.

12. Brasil. Resolução COFFITO no 80. Baixa Atos Complementares à Resolução COFFITO no 08, relativa ao exercício profissional do fisioterapeuta, e à Resolução COFFITO no 37, relativa ao registro de empresas nos conselhos regionais de Fisioterapia e Terapia Ocupacional, e dá outras providências. Diário Oficial da União 1987; 21 mai.

13. Bodstein R, Ramos CL, Hortale VA. Programa de reabilitação comunitária. Experiência de inclusão social da pessoa portadora de deficiência FU N LAR - Rio de Janeiro. Rio de Janeiro: Fundação Oswaldo Cruz; 2003.
14. Nogueira JA. Fisioterapia no contexto da assistência domiciliar terapêutica: estudos de pacientes com AIDS [dissertação]. Rio de Janeiro (RJ): Escola Nacional de Saúde Pública Sérgio Arouca, Fundação Oswaldo Cruz; 2000.

15. Teixeira CF. Modelos de atenção voltados para a qualidade, efetividade, equidade e necessidades prioritárias de saúde. In: Cadernos da 11ạ Conferência Nacional de Saúde. Brasília: M inistério da Saúde; 2000.

16. Mendes EV. A Atenção primária à saúde no SUS. Fortaleza: Escola de Saúde Pública do Ceará; 2002.

17. Brasil. M inistério da Saúde. Política Nacional de Atenção Básica. Brasília: M inistério da Saúde; 2006.

18. Sousa M F. A Cor-Agem do PSF. São Paulo: Hucitec; 2003.

19. Reis CCL. Supervisão no PSF - Limites e possibilidades [dissertação]. Rio de Janeiro (RJ): Escola Nacional de Saúde Pública Sérgio Arouca, Fundação Oswaldo Cruz; 2001.

20. Moreira MR, Cruz N eto O, Sucena LFM. A relação dos profissionais do Programa Saúde da Família com o contexto de exclusão social da população atendida. Revista Saúde em Foco 2002; 23:65-81.

21. Cordeiro H. Os desafios do ensino das profissões da saúde diante das mudanças do modelo assistencial: contribuição para além dos pólos de capacitação em Saúde da Família. Revista Divulgação em Saúde para Debate 2000; 21.

22. Merhy EE. Um ensaio sobre o médico e suas valises tecnológicas. Contribuições para compreender as reestruturações produtivas do setor saúde. Interface (Botucatu) 2000; 6:109-116.

Artigo apresentado em 14/08/2007

Versão final apresentada em 14/12/2007 